\title{
Pattern of Sleep in Infants and Toddlers Visiting at a Teaching Hospital in Lalitpur
}

\author{
Shrestha $D^{1}$, Dhakal AK², Shakya $A^{3}$, Shah SC $^{4}$, Shakya $\mathrm{H}^{5}$, Mehata $\mathrm{S}^{6}$, Sadeh $\mathrm{A}^{7}$
}

\begin{abstract}
Introduction: Sleep is an integral part in a child's health and development. During different stages of development, there are aberrations in normal physiology of sleep which make children more susceptible to various types of sleep problems. This study was conducted to identify sleep pattern and sleep problems in Nepalese children using Nepali translation of Brief Infant Sleep Questionnaire (BISQ) screening tool. Materials and Methods: This was a descriptive cross-sectional study. Data were collected from parents of children aged 6 to 36 months attending paediatric out-patient clinic for general health check-up from July 2013 to December 2013. Nepali translation of the BISQ was used as a screening tool for sleep problems in this study. Results: The study included 553 children ( $52.4 \%$ boys). Mothers completed the questionnaire in $92 \%$ of children. Mean total duration of sleep in 24 hours was $11.7 \pm 1.7$ hours. Respondents reported sleep problem in $16.9 \%$ of children whereas $20.3 \%$ of children had sleep problems according to BISQ criteria. As per this criteria, $2.7 \%$ of children had total sleep duration less than 9 hours, $5.9 \%$ of children had total night awakening duration of more than one hour, $13.9 \%$ of children had night waking $>3$ times. These BISQ sleep parameters differed significantly in children with and without sleep problems $(p<0.001)$. In addition, children with sleep problem took longer time to sleep than children without sleep problems $(p<0.001)$. Conclusion: Sleep problems are common in Nepalese infants and toddlers according to the BISQ. Screening for sleep problems is highly recommended during health care visits given the prevalence of sleep problems identified by this study to reduce their potential adverse impact.
\end{abstract}

Key words: BISQ, Infant, Nepal, Sleep, Sleep problem, Sleep questionnaire.

\section{Introduction}

leep is a basic biologic function of a child and constitutes a significant proportion of life. Sleep pattern varies with age and plays a vital role in a child's growth and development. In addition, sleep is strongly influenced by environmental and cultural factors 1,2 . Hence normal sleep patterns should be defined based on child's developmental, sociocultural and environmental factors. Sleep
'Dr. Devendra Shrestha, Associate Professor, Department of Paediatrics, KIST Medical College and Teaching Hospital, Imadol, Lalitpur, Nepal, ${ }^{2}$ Dr. Ajaya Kumar Dhakal, Lecturer, Department of Paediatrics, KIST Medical College and Teaching Hospital, Imadol, Lalitpur, Nepal, ${ }^{3}$ Dr. Arati Shakya, Lecturer, Department of Paediatrics, KIST Medical College and Teaching Hospital, Imadol, Lalitpur, Nepal, ${ }^{4}$ Dr. Subhash Chandra Shah, Lecturer, Department of Paediatrics, KIST Medical College and Teaching Hospital, Imadol, Lalitpur, Nepal. ${ }^{5}$ Dr. Henish Shakya, Lecturer, Department of Paediatrics, KIST Medical College and Teaching Hospital, Imadol, Lalitpur, Nepal. ${ }^{6} \mathrm{Dr}$. Suresh Mehata, MPH, PhD, Research Advisor, Nepal Health Sector Support Programme (NHSSP), Ministry of Health and Population, Ram Shah Path, Nepal, ${ }^{7}$ Dr. Avi Sadeh, DSc, Professor, School of Psychological Sciences, Tel Aviv University, Tel Aviv, Israel.

\author{
Address for correspondence: \\ Dr. Devendra Shrestha \\ Department of Paediatrics, \\ KIST Medical College and Teaching Hospital, \\ Imadol, Lalitpur, Nepal. \\ Email: devendra.shrestha@gmail.com \\ Tel No; +977-9851108123
}

\section{How to cite}

Shrestha D, Dhakal AK, Shakya A, Shah SC, Shakya $H$, Mehata S, Sadeh A. Pattern of Sleep in Infants and Toddlers Visiting at a Teaching Hospital in Lalitpur. J Nepal Paediatr Soc 2015;35(1):24-30.

doi: http://dx.doi.org/10.3126/jnps.v35i1.12093

This work is licensed under a Creative Commons Attribution 3.0 License.

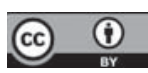

pattern of children as well as parental perception to sleep problem differ in Asian countries from the western countries ${ }^{3}$. These factors should be identified for understanding sleep problems. 
Sleep problems are also associated with various other problems like learning disabilities, impaired cognitive functions, behavioural disorders, and emotional problems ${ }^{4}$. Although maturation leads to improvement in some aspects of sleep pattern, sleep problems in early childhood are likely to persist to later years ${ }^{5}$.

Sleep problems are common in up to $25 \%$ of children ${ }^{2}$. Yet, these problems are still under-recognized and neglected by physicians and parents in common practice $^{6}$.

The English version of Brief Infant Sleep Questionnaire (BISQ) is one of the screening tools used to identify sleep problems in children below 36 months age $^{7}$ and which has been used in various studies worldwide ${ }^{8,9}$. It is a simple tool and constitutes of 11 questions and can be completed by responders within 5 to 10 minutes.

Currently there is a lack of data regarding sleep behaviour and sleep related problems in young Nepalese children. Therefore this study was conducted to identify various patterns of sleep and, common sleep problems among infants and toddlers in Nepal using BISQ. In addition to documenting the prevalence of sleep problem in Nepal, the findings could be compared to normative data of children from different parts of world.

\section{Materials and Methods}

This was a descriptive cross-sectional study conducted among children aged six months to thirty six months attending paediatric out-patient clinic for general health check-up from July 2013 to December 2013. Ethical clearance was obtained from institutional review committee prior conducting the study. Parents or caregivers were asked to complete the translated Nepali translation of BISQ after an informed written consent. Children with any acute or severe medical problems or on stimulant medications potentially affecting sleep were excluded from the study.

The BISQ is a screening tool to detect common sleep problems in infants and toddlers ${ }^{7}$. This questionnaire was translated in Nepali language using standard technique and details have been described earlier ${ }^{10}$. This Nepali translated version the BISQ was used in the current study.

Children were screened by one of the researchers for any acute illness or medical problems and parents were asked to fill up the Nepali version of BISQ. In case, attending guardian was illiterate, an independent person was asked to read the questions to the guardian and complete the questionnaire.

We defined a sleep problem if (a) three or more night awakenings were reported; (b) duration of nighttime awakening was more than 1 hour; or (c) the total duration of sleep was less than 9 hours ${ }^{7}$.

Data was analyzed with SPSS 16.0, using two independent sample t-test for normally distributed numerical variables and Mann-Whitney $U$ test for nonnormally distributed numerical variables and binomial logistic regression for categorical data to assess the association between various sleep parameters and $p<0.05$ was considered statistically significant.

\section{Results}

The survey was successfully completed in 553 children attending paediatric out-patient clinic for general health services. Out of which, 290 (52.4\%) children were boys (Table 1). Mothers completed the questionnaire in 510 (92.2\%) children. Mean age of children was $18.9 \pm 8.8$ months.

Sleep characteristics are summarized in Table 2 . Almost all (99.3\%) children slept in the same room with either parents or grandparents whereas bed sharing with parents or grandparents was seen in $93 \%$ of children. Most of children preferred lateral position during sleep and almost half of children required feeding to fall asleep.

Other sleep parameters are presented in Table 3. Overall median sleep time was 8:30 PM and mean total sleep duration was $11.7 \pm 1.7$ hours which decreased with increasing age. However when analyzed sleep time in day and night, mean night time sleep duration increased and mean day time sleep duration decreased gradually with increasing age.

Among children who met our criteria for a sleep problem according to the BISQ, $2.7 \%$ had total sleep duration less than nine hours, $5.9 \%$ had night waking duration of more than one hour and $13.9 \%$ had night waking more than three times in a night. Significant differences were noted between the BISQ measures in respondents who self-identified sleep problem and those who did not: mean total sleep duration in hours $(11.0 \pm 1.8$ versus $11.8 \pm 1.7, p<0.001)$, mean night waking duration in minutes $(45.0 \pm 56.8$ versus $19.0 \pm 23.1, p<0.001)$, mean number of night waking (2.6 \pm 1.6 versus $2.1 \pm 1.4, p<0.001$ ). 
Table 1: Baseline characteristics of children

\begin{tabular}{|c|c|c|c|}
\hline & & Number & Percentage \\
\hline \multirow{3}{*}{ Age group } & $6-12$ months & 177 & 32.0 \\
\cline { 2 - 4 } & $13-24$ months & 225 & 40.7 \\
\cline { 2 - 4 } & $25-36$ months & 151 & 27.3 \\
\hline \multirow{3}{*}{ Sex } & Male & 290 & 52.4 \\
\cline { 2 - 4 } & Female & 263 & 47.6 \\
\hline \multirow{3}{*}{ Birth order } & Eldest & 370 & 66.9 \\
\cline { 2 - 4 } & Middle & 21 & 3.8 \\
\cline { 2 - 4 } & Youngest & 162 & 29.3 \\
\cline { 2 - 4 } & Mother & 510 & 92.2 \\
\hline \multirow{3}{*}{ Responder } & Father & 30 & 5.4 \\
\hline
\end{tabular}

Table 2: Sleep characteristics of children

\begin{tabular}{|l|c|c|c|}
\hline \multirow{4}{*}{ Place of sleep } & Same bed as parents & Number & Percentage \\
\hline \multirow{3}{*}{ Sleeping position } & Small cot/crib in same room as parents & 511 & 92.4 \\
\cline { 2 - 4 } & $\begin{array}{c}\text { Small bed/crib in a separate room with } \\
\text { other sibling }\end{array}$ & 35 & 6.3 \\
\cline { 2 - 4 } & Same bed of grandparents & 4 & 0.7 \\
\cline { 2 - 4 } & Lateral & 3 & 0.5 \\
\hline \multirow{3}{*}{ Bed time rituals } & Supine & 288 & 52.1 \\
\cline { 2 - 4 } & Prone & 191 & 34.5 \\
\cline { 2 - 4 } & While feeding & 74 & 13.4 \\
\cline { 2 - 4 } & Rocking & 85 & 48.8 \\
\cline { 2 - 4 } & Lying in bed alone & 77 & 15.4 \\
\cline { 2 - 4 } & Lying on bed along with parents & 77 & 13.9 \\
\hline
\end{tabular}

Table 3: Sleep parameters according to age group

\begin{tabular}{|l|c|c|c|c|c|}
\hline & Overall & $\mathbf{6 - 1 2}$ months & $\mathbf{1 3 - 2 4}$ months & $\mathbf{2 5 - 3 6}$ months & p-value \\
\hline Mean total sleep duration (Hours) & $11.7 \pm 1.7$ & $12.0 \pm 1.8$ & $11.6 \pm 1.7$ & $11.5 \pm 1.7$ & 0.027 \\
\hline Mean night sleep duration (Hours) & $9.4 \pm 1.2$ & $9.3 \pm 1.2$ & $9.3 \pm 1.2$ & $9.7 \pm 1.1$ & 0.001 \\
\hline Mean day sleep duration (Hours) & $2.3 \pm 1.3$ & $2.7 \pm 1.3$ & $2.3 \pm 1.2$ & $1.7 \pm 1.2$ & $<0.001$ \\
\hline Mean time to put to sleep (Minutes) & $20.9 \pm 20.2$ & $20.1 \pm 18.6$ & $21.7 \pm 20.2$ & $20.5 \pm 22.0$ & 0.728 \\
\hline $\begin{array}{l}\text { Mean duration of night waking } \\
\text { (Minutes) }\end{array}$ & $23.5 \pm 33.0$ & $27.2 \pm 30.6$ & $25.1 \pm 37.7$ & $16.6 \pm 26.4$ & 0.009 \\
\hline Mean number of night waking & $2.2 \pm 1.4$ & $2.5 \pm 1.3$ & $2.4 \pm 1.4$ & $1.4 \pm 1.4$ & $<0.001$ \\
\hline
\end{tabular}

Table 4: Sleep characteristics of children according to sleep problems perceived by respondents

\begin{tabular}{|l|c|c|c|c|}
\hline & Major problem & Small problem & No problem & $\boldsymbol{p}$-value \\
\hline Mean total sleep duration (Hours) & $10.7 \pm 1.1$ & $11.1 \pm 1.9$ & $11.8 \pm 1.7$ & $<0.001$ \\
\hline Mean night sleep duration (Hours) & $8.6 \pm 1.1$ & $8.8 \pm 1.2$ & $9.5 \pm 1.2$ & $<0.001$ \\
\hline Mean day sleep duration (Hours) & $2.1 \pm 0.8$ & $2.3 \pm 1.3$ & $2.3 \pm 1.3$ & 0.802 \\
\hline Mean time to put to sleep (Minutes) & $41.1 \pm 46.3$ & $30.2 \pm 24.5$ & $18.5 \pm 16.8$ & $<0.001$ \\
\hline Mean duration of night waking (Minutes) & $83.5 \pm 97.4$ & $36.5 \pm 39.4$ & $19.0 \pm 23.1$ & $<0.001$ \\
\hline Mean number of night waking (Times per night) & $3.5 \pm 2.3$ & $2.5 \pm 1.4$ & $2.1 \pm 1.4$ & $<0.001$ \\
\hline
\end{tabular}


Table 5: Proportion of children with sleep problems (as defined by BISQ) according to age groups

\begin{tabular}{|l|c|c|c|c|}
\hline BISQ parameter & $\begin{array}{c}\mathbf{6 - 1 2} \text { months } \\
(\mathbf{n = 1 7 7 )}\end{array}$ & $\begin{array}{c}\mathbf{1 3 - 2 4} \text { months } \\
(\mathbf{n = 2 2 5})\end{array}$ & $\begin{array}{c}\mathbf{2 5 - 3 6} \text { months } \\
(\mathbf{n = 1 5 1 )}\end{array}$ & $\begin{array}{c}\text { Total } \\
(\mathbf{n}=553)\end{array}$ \\
\hline Sleep duration $<9$ hours & $2(1.1 \%)$ & $8(3.6 \%)$ & $5(3.3 \%)$ & $15(2.7 \%)$ \\
\hline Number of night waking $>3$ times & $36(20.3 \%)$ & $33(14.7 \%)$ & $8(5.3 \%)$ & $77(13.9 \%)$ \\
\hline Duration of night waking $>1$ hour & $12(6.8 \%)$ & $15(6.7 \%)$ & $6(4 \%)$ & $33(5.9 \%)$ \\
\hline
\end{tabular}

Table 6: Parent reported sleep problems

\begin{tabular}{|l|c|c|}
\hline Problem & Number $(\mathbf{n = 9 4 )}$ & Percentage \\
\hline Inadequate sleep & 20 & 21.3 \\
\hline Difficult to put sleep & 19 & 20.2 \\
\hline Frequent night waking & 18 & 9.5 \\
\hline Sleep position & 9 & 7.4 \\
\hline Cries during sleep & 7 & 6.4 \\
\hline Wakes for a long time during night & 6 & 5.3 \\
\hline Moves a lot during sleep & 5 & 4.3 \\
\hline Seeks feed during sleep & 4 & 3.2 \\
\hline Snores during sleep & 3 & 1.1 \\
\hline Sleeps late at night & 1 & 1.1 \\
\hline Finger sucking during sleep & 1 & 1.1 \\
\hline Bed wetting & 1 & \\
\hline
\end{tabular}

When we compared children with or without sleep problem as defined by BISQ criteria, mean day time sleep duration was not statistically different between these two groups of children however, total sleep duration and night time sleep duration were significantly different between children with sleep problem and without sleep problem (total sleep duration $11.1 \pm 1.9$ hours versus $11.8 \pm 1.7$ hours, $p<0.001$; night sleep duration $8.8 \pm 1.3$ hours versus $9.6 \pm 1.1$ hours, $p<0.001$ ). Children with sleep problem had mean number of night awakenings of $3.9 \pm 1.5$ per night as compared to $1.7 \pm 1$ per night in children without sleep problem. Similarly duration of night waking was significantly higher in children with sleep problem (55.5 \pm 55.7 minutes) than children without sleep problem $(15.3 \pm 15.8, p<0.001)$.

Children fell asleep in mean duration of 20.9 minutes. Though not included in BISQ criteria, time to fall asleep was found to be statistically significant (28.6 \pm 28.6 minutes in children with sleep problem versus $18.9 \pm 16.9$ minutes in those without sleep problem, $p<0.001)$. Median time of sleep time was 9.00 PM and 8.30 PM respectively in children with sleep problem and without sleep problem as categorized by BISQ.

Sleep problems were perceived by 94 (16.9\%) responders in their children; out of whom 17 (3\%) responders considered as major problem and 77 (13.9\%) responders as a small problem. Sleep parameters of these children are shown in Table 4. However applying the BISQ criteria, 112 (20.3\%) children were found to have sleep problems. Compared to BISQ, parental reported sleep problem were: true positive $32.1 \%$, true negative $86.8 \%$, false positive $13.2 \%$ and false negative $67.9 \%, p<0.001$.

Similarly, sleep problems were observed in $25.4 \%$, $21.8 \%$ and $11.9 \%$ children in age groups $6-12$ months, 13-24 months and 25-36 months respectively which was statistically significant $(p=0.006)$.

There was no significant association of sleep problem with place and position of sleep, bed time rituals or birth order.

Parent perceptions of sleep problems in their children are shown in Table 6 . Inadequate sleep, bed time resistance and night awakening were the common parental concerns. Interestingly sleeping in prone or lateral or supine position was considered as a problem by few parents.

\section{Discussion}

Normal sleep pattern in infants and toddlers is influenced by age, socio-cultural practices and the 
environment in which a child grows in interrelationship with other developmental processes. The perception of parents regarding sleep pattern of their children is also determined by socio-cultural practices dealing with sleep and education related to sleep hygiene and is different worldwide. Therefore, sleep problems in children may occur as a result of derangement in any of these multiple factors.

This study describes the presence of sleep problems in Nepalese children. Sleep problems were identified in $20.3 \%$ infants and toddlers based on BISQ criteria which was lower than that observed (33\%) in another study in Indonesia using the same criteria ${ }^{8}$.

Co-sleeping of children with parents is a common and acceptable practice as it is considered unreasonable to put children to sleep alone in various communities. In Nepalese society, as in other Asian countries ${ }^{12}$, co-sleeping up to a certain age is also a cultural norm and a separate bed room for children is not common. The same issue was reflected in this study where $92 \%$ of children co-slept with parents, similar to children in countries predominant Asian (64.5\%) ${ }^{3}$, India $(96 \%)^{13}$ and Indonesia $(99.3 \%)^{8}$. However, bed sharing with parents was very low (7.2 to 16.6\%) in European countries and Australia ${ }^{5,9}$. Co-sleeping per se was not found to be associated with increased sleep problems in early childhood in previous studies ${ }^{14}$. However trying to get children asleep and subsequently falling asleep at a similar time may disturb parents' sleep quality, which may subsequently affect that of their children ${ }^{14}$.

Bed time rituals also differed between Asian and Caucasian children. Most Caucasian children (57\%) were put to sleep independently in their own crib or bed compared to $4 \%$ in Asian children ${ }^{15}$. In Asian children, feeding was observed as the most common bed-time ritual in $60 \%$ of children ${ }^{8}$. There was a high level of parental involvement in sleep onset as well as maintenance of sleep in children in the present study similar to observed among children in Asian countries ${ }^{15}$. Feeding was the most preferred method of sleep initiation among Nepalese children in this study while co-sleeping was most common method of maintaining sleep.

Sleep-wake regulation and its maintenance is a developmental process which matures rapidly during infancy and early childhood and the process continues throughout the childhood period. Circadian rhythm of sleep develops during early infancy and daytime napping continues till four years of age ${ }^{16}$. Similarly night awakenings are frequent in infancy and may be as high as $30 \%$ which gradually decrease over the age ${ }^{17}$. Difficulties in maturation of sleep are clinically evident in the total very short sleep episodes and in multiple and extended night awakenings, which has an inverse relation to age $\mathrm{e}^{18}$. This normal physiological pattern of decreasing total duration of sleep and duration of night awakening according to age was also reflected in this study.

A systematic review has described total sleep duration of 12.7 hours during infancy, 12.6 hours at one to two years and 12 hours at two to three years of age ${ }^{18}$. However total sleep duration was shorter in Asian children (12.31 hours) in comparison to western countries (13.02 hours) $)^{3}$. Total sleep duration of children in the current study was also within the lower range of sleep duration among children of primarily Asian origin and was similar to lowest duration which was observed among children in Japan (11.6 hours) ${ }^{3}$.

A large study among children aged below three years showed Asian children (9.44 PM) have significantly late bed time than Caucasian children (8.42 PM) ${ }^{3}$. This was also reflected in the current study as the median sleep time of 9.15 PM in our children.

This study showed higher rate of night awakenings as compared to other countries. This may be explained with the finding that almost all of children slept with parent and parent will be more aware of these night awakenings. Mean time to put to sleep was 20 minutes in Asian children ${ }^{8}$, which was similar to this study. In a systemic review of children below two years of age sleep latency was found to be 19 minutes $^{18}$. Sleep latency is an important indicator because many parents consider small duration of sleep latency as the most important part of healthy sleep and difficulty putting their children to sleep as a worrisome sign. However, it is important to remember parental reporting of sleep latency time may be exaggerated as compared to latency time measured by polysomnography ${ }^{19}$.

The factors most concerning to parents in this study were longer time to get asleep (sleep onset latency), frequent night awakening (poor sleep maintenance) and shorter sleep duration, which were in concordance to other studies ${ }^{5,13,17}$.

Parental perception to sleep problems vary across different cultures and age groups and parents of Asian origin are more likely to perceive problem in their children's sleep ${ }^{3}$. Parental perception as sleep problem varied from $11 \%$ to $76 \%$ in studies from Asian countries $^{3}$. Whereas in an Indonesian study using 
BISQ, $15.7 \%$ of parents perceived that their children have sleep problem ${ }^{8}$. In contrast, parental perception as sleep problem was seen only in $10 \%$ in developed countries from Europe and United States ${ }^{5}$. These data along with our study suggest that caution must be exercised when eliciting history regarding parentdefined sleep problem in children especially from parents from Asian background. Therefore it is rational to screen children using structured sleep questionnaire rather than relying on parental perception to detect sleep problems and followed by detailed evaluation if needed.

This study highlights the existence of sleep problems in Nepalese children as well as limitations of parental perception of sleep problem. In general, there is lack of importance given to sleep problems by caregivers as well as even health care providers who may miss identification of sleep problems in a child ${ }^{5}$. Hence the use of structured screening questionnaires during hospital visits is recommended to screen for sleep problems and those children with sleep problem should further be evaluated in detail and appropriately intervened in presence of sleep problems.

Reporter bias and inapplicability in children with developmental disorders like autism are the limitations of this study. Similarly caution should be exercised to generalize this data to whole of Nepalese children as this was conducted among apparently well children visiting a hospital in a semi-urban community.

\section{Conclusions}

Most infants and toddlers slept in same bed with parents. Most children were dependent on parents for the initiation as well as maintenance of their sleep. Nepali translation of BISQ may be used to screen for sleep problems. As in other parts of world, sleep problem was common in Nepalese children. Health care providers should be aware of existence of sleep problems in Nepalese children and hence it is imperative to screen children during hospital visits by using screening tools. Further large scale population based studies will be able to ascertain an exact incidence of sleep problem in Nepal. In addition, studies exploring the nature of these sleep problems are required.

Acknowledgement: None

Funding: None

Conflicts of Interest: None

Permission from IRB: Yes

\section{References}

1. Jenni OG, O'Connor BB. Children's sleep: an interplay between culture and biology. Pediatrics 2005;115(1 Suppl):204-16.

2. Owens JA. Introduction: Culture and sleep in children. Pediatrics 2005;115(1 Suppl):201-3.

3. Mindell JA, Sadeh A, Wiegand B, How TH, Goh DY. Cross-cultural differences in infant and toddler sleep. Sleep Med 2010;11(3):274-80.

4. Lewandowski AS, Toliver-Sokol M, Palermo TM. Evidence-based review of subjective pediatric sleep measures. J Pediatr Psychol 2011;36(7):78093.

5. Byars KC, Yolton K, Rausch J, Lanphear B, Beebe DW. Prevalence, patterns, and persistence of sleep problems in the first 3 years of life. Pediatrics 2012;129(2):e276-84.

6. Adam EK, Snell EK, Pendry P. Sleep timing and quantity in ecological and family context: a nationally representative time-diary study. J Fam Psychol 2007;21(1):4-19.

7. Sadeh A. A Brief Screening Questionnaire for Infant Sleep Problems: Validation and findings for an internet sample. Pediatrics 2004;113(6):e570-e7.

8. Sambo CM, Sekartini R, Trihono PP. Sleep patterns in 1 to 36 month-old children. Paediatr Indones 2010;50(3):170-5.

9. Teng A, Bartle A, Sadeh A, Mindell J. Infant and toddler sleep in Australia and New Zealand. J Paediatr Child Health 2012;48(3):268-73.

10. Dhakal AK, Shrestha D, Shah SC, Shakya H, Shakya A, Sadeh A. A Nepali translation of Brief Infant Sleep Questionnaire (BISQ) for assessment of sleep in infants and toddlers: a preliminary report. Journal of Kathmandu Medical College 2014;3(3) (9):102-6.

11. Sadeh A, Mindell J, Rivera L. "My child has a sleep problem": a cross-cultural comparison of parental definitions. Sleep Med 2011;12(5):478-82.

12. Anuntaseree W, Mo-Suwan L, Vasiknanonte P, Kuasirikul S, Ma-A-Lee A, Choprapawon C. Factors associated with bed sharing and sleep position in Thai neonates. Child Care Health Dev 2008;34(4):482-90.

13. Ravikiran SR, Kumar PM, Latha KS. Sleep problems in preschool and school aged rural Indian children. Indian Pediatr 2011;48(3):221-3.

14. Latz S, Wolf AW, Lozoff B. Cosleeping in context: sleep practices and problems in young children in 
Japan and the United States. Arch Pediatr Adolesc Med 1999;153(4):339-46.

15. Mindell JA, Sadeh A, Kohyama J, How TH. Parental behaviors and sleep outcomes in infants and toddlers: a cross-cultural comparison. Sleep Med 2010;11(4):393-9.

16. Iglowstein I, Jenni OG, Molinari L, Largo RH. Sleep duration from infancy to adolescence: reference values and generational trends. Pediatrics 2003;111(2):302-7.

17. Thunstrom M. Severe sleep problems among infants in a normal population in Sweden: prevalence, severity and correlates. Acta Paediatr 1999;88(12):1356-63.

18. Galland BC, Taylor BJ, Elder DE, Herbison P. Normal sleep patterns in infants and children: a systematic review of observational studies. Sleep Med Rev 2012;16(3):213-22.

19. Goodwin JL, Silva GE, Kaemingk KL, Sherrill DL, Morgan WJ, Quan SF. Comparison between reported and recorded total sleep time and sleep latency in 6- to 11-year-old children: the Tucson Children's Assessment of Sleep Apnea Study (TuCASA). Sleep Breath 2007;11(2):85-92. 